INPLASY

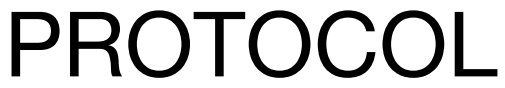

To cite: Jiang et al. Risk factors for autism spectrum disorder gastrointestinal symptom:an overview of reviews. Inplasy protocol 202210134. doi: 10.37766/inplasy2022.1.0134

Received: 30 January 2022

Published: 30 January 2022

Corresponding author:

Yuwei Jiang

15704601990@163.com

Author Affiliation:

Jiamusi University.

Support: Jiamusi Doctoral Fund.

Review Stage at time of this submission: Preliminary searches.

Conflicts of interest: None declared.

\section{Risk factors for autism spectrum disorder gastrointestinal symptom: an overview of reviews}

Review question / Objective: Search risk factors for autism spectrum disorder gastrointestinal symptom.

Condition being studied: According to the World Health Organization, approximately 1 in 160 children worldwide have ASD. In the United States, the current incidence of ASD is about 1 in 54 children. Related research results also found that 26.2 percent of Autistic Children in China reported gastrointestinal issues, more than double the prevalence in the usual development comparison group.

Information sources: The following databases were searched from inception until December 2021:EMBASE, The Cochrane Library, PubMed, Web of Science and Scopus. The search process for this umbrella review included the following search terms, combinations of terms and their derivatives: ((autism)or(pervasive developmental disorder) or(Asperger)) and(systematic review)or (meta-analysis). To combine the keywords, Boolean operators "AND" and "OR" were applied. No limiters were applied. In addition, the references of the reviews identified for inclusion were examined for any further systematic reviews not found in the initial search of the electronic databases.

INPLASY registration number: This protocol was registered with the International Platform of Registered Systematic Review and Meta-Analysis Protocols (INPLASY) on 30 January 2022 and was last updated on 30 January 2022 (registration number INPLASY202210134).

\section{INTRODUCTION}

Review question / Objective: Search risk factors for autism spectrum disorder gastrointestinal symptom.
Condition being studied: According to the World Health Organization, approximately 1 in 160 children worldwide have ASD.In the United States, the current incidence of ASD is about 1 in 54 children.Related research 
results also found that 26.2 percent of Autistic Children in China reported gastrointestinal issues, more than double the prevalence in the usual development comparison group.

\section{METHODS}

Participant or population: Autism with gastrointestinal symptoms will be included.

Intervention: Any factors affecting gastrointestinal function in autistic patients will be included.

Comparator: Any factors affecting gastrointestinal function in autistic patients will be included.

Study designs to be included: (systematic review)or (meta-analysis).

Eligibility criteria: The search titles were uploaded in Endnote software and duplicates removed. One author screened the papers, first the titles and abstracts and when required, the full text. $A$ second author checked the screening. Disagreements were planned to be adjudicated by a third author. A PRISMA flow diagram of the papers screened, included, and excluded was generated.

Information sources: The following databases were searched from inception until December 2021:EMBASE, The Cochrane Library, PubMed, Web of Science and Scopus. The search process for this umbrella review included the following search terms, combinations of terms and their derivatives: ((autism)or(pervasive developmental disorder) or(Asperger)) and(systematic review)or (meta-analysis). To combine the keywords, Boolean operators "AND" and "OR" were applied. No limiters were applied. In addition, the references of the reviews identified for inclusion were examined for any further systematic reviews not found in the initial search of the electronic databases.

Main outcome(s): Any factors affecting gastrointestinal function in autistic patients will be included.
Quality assessment / Risk of bias analysis: Any factors affecting gastrointestinal function in autistic patients will be included.

Strategy of data synthesis: Any factors affecting gastrointestinal function in autistic patients will be included.

Subgroup analysis: The current query no subgroups

Sensitivity analysis: Sensitivity analysis involved visual inspection of Cls for the overall ES after each study was removed one at a time. No study significantly altered the overall mean ES estimates for each of the $\mathrm{GI}$ variables included in the analysis.

Country(ies) involved: China.

Keywords: gastrointestinal symptoms; meta analysis; diet.

Contributions of each author:

Author 1 - Yuwei Jiang.

Email: 15704601990@163.com

Author 2 - Wenjun Dang.

Email: 304532709@qq.com

Author 3 - Xiangying Kong.

Email: 15663960058@163.com

Author 4 - Jin Guo.

Email: 13089827667@163.com

Author 5 - Hong Nie.

Email: 18903648756@163.com 